\section{Measuring the radius of curvature of a spheri- cal mirror with an interferometer and a laser tracker}

\author{
Chunyu Zhao, MEMBER SPIE \\ Rene Zehnder, MEMBER SPIE \\ James H. Burge, FELLOW SPIE \\ University of Arizona \\ College of Optical Sciences \\ 1630 E. University Blvd. \\ Tucson, Arizona 85721 \\ E-mail: czhao@optics.arizona.edu
}

Abstract. We present a method to accurately measure the radius of curvature of a concave spherical mirror with a phase-measuring interferometer and a laser tracker. Use of a laser tracker eases the alignment of the testing system, eliminates the need to move the test piece during the measurement, and improves the accuracy of the distance measurement. Using this method, we measured the radius of curvature of a spherical mirror $0.5 \mathrm{~m}$ in diameter and about $2.5 \mathrm{~m}$ in radius of curvature. The accuracy of the measurement is better than $\pm 20 \mu \mathrm{m}$. ( ) 2005 Society of Photo-Optical Instrumentation Engineers.

[DOI: 10.1117/1.2047594]

Subject terms: metrology; radius of curvature; laser tracker.

Paper 050170 LR received Mar. 8, 2005; revised manuscript received Jun. 27, 2005; accepted for publication Jul. 14, 2005; appeared online Jul. 14, 2005; published online Sep. 14, 2005.

\section{Introduction}

Accurate measurement of the radius of curvature (ROC) of a spherical mirror is often critical to the performance of the optical system where the mirror is used. A variety of ways exists to measure the ROC of a spherical mirror. ${ }^{1-5}$ The majority of them can be classified into two categories: direct and indirect methods. ${ }^{2}$ In direct methods, the center of curvature (COC) of the test surface is located, and the distance from it to the surface is measured by either mechanical or optical means. ${ }^{3}$ In indirect methods, surface sag is measured and the ROC is then calculated from the sag value. Both methods have drawbacks. The direct method requires a large work space for long radii and is susceptible to distance measurement errors, while the indirect method suffers the extreme sensitivity to sag measurement errors. For accurate ROC measurements, a direct method employing a phase-measuring interferometer (PMI) and a distancemeasuring interferometer (DMI) is often used. ${ }^{3,4}$ The test surface is measured at the cat's-eye position first and then moved to the confocal position. When the cat's-eye and the $\mathrm{COC}$ are not perfectly aligned with the laser beam focus, the PMI sees no perfect nulls. The longitudinal offsets of the cat's-eye and the COC from the laser beam focus are defined as null cavity errors, ${ }^{3}$ which can be calculated from the wavefront errors the PMI measures at each position and

0091-3286/2005/\$22.00 @ 2005 SPIE compensated in the calculation of the ROC. Two drawbacks are associated with this method. First, the test piece must be moved from the cat's-eye position to the confocal position. This is rather inconvenient when the test piece is large and heavy. Second, the PMI measurement at the cat's-eye position is insensitive to the test surface tilt and decenter, ${ }^{4}$ which makes it difficult to achieve high accuracy in distance measurement by a DMI. In this letter, we present a method to use a laser tracker (see, e.g., Ref. 6) with a PMI to perform ROC measurement on spherical mirrors. Replacement of a DMI with a laser tracker eliminates the aforementioned drawbacks, and high measurement accuracy is achieved.

\section{Method Description}

A laser tracker is essentially a coordinate measurement machine (CMM). It measures polar coordinates instead of Cartesian coordinates. A laser beam is sent out and reflected back by a sphere-mounted retroreflector (SMR). When the SMR is moved, a feed back sensor detects the motion and keeps tracking it. A laser tracker uses a DMI to measure the distance, and two angular encoders to measure the rotation angles. So the laser tracker can be used as a DMI to measure the ROC of a spherical mirror with a PMI, yet its tracking ability makes it much easier to align than a standalone DMI. More importantly, compared to the method using a PMI and a DMI, this method does not require the test surface to be moved. Instead, the test surface is fixed at the confocal position with its COC nearly coincident with the focus of the PMI's laser beam, and the SMR is moved from the PMI beam focus to the test surface while the laser tracker measures the distance it moves. Since the laser tracker measures distances in 3-D space, we have more freedom to place it wherever convenient. In fact, when the laser tracker is put next to the PMI and close to the laser beam focus, the ROC measurement has higher accuracy and the entire testing system is more compact. Figure 1 illustrates the setup of such a measurement system.

The measurement procedure is summarized here:

1. Set up the PMI and the spherical mirror such that the PMI beam focus coincides as closely as possible with the COC of the mirror. Put the laser tracker ball (SMR) at the beam focus with the retroreflector facing the laser tracker and the reflecting spherical surface facing the PMI. From the power term in the measured wavefront, we can calculate the longitudinal defocus $\Delta 1$ (null cavity error).

2. Use the laser tracker to get the position of the tracker ball at the laser beam focus.

3. Move the tracker ball to touch the spherical surface, and get its position again. The distance $L$ between the two SMR positions can then be obtained from the laser tracker.

4. The PMI is looking at the surface of the test mirror. Measure the wavefront. From the power term in the wavefront error, we can calculate the longitudinal defocus $\Delta 2$ (null cavity error).

5. The radius of curvature of the spherical mirror can then be calculated: $R=L+\Delta 1+\Delta 2+r$, where $r$ is the $\mathrm{ROC}$ of the tracker ball. 


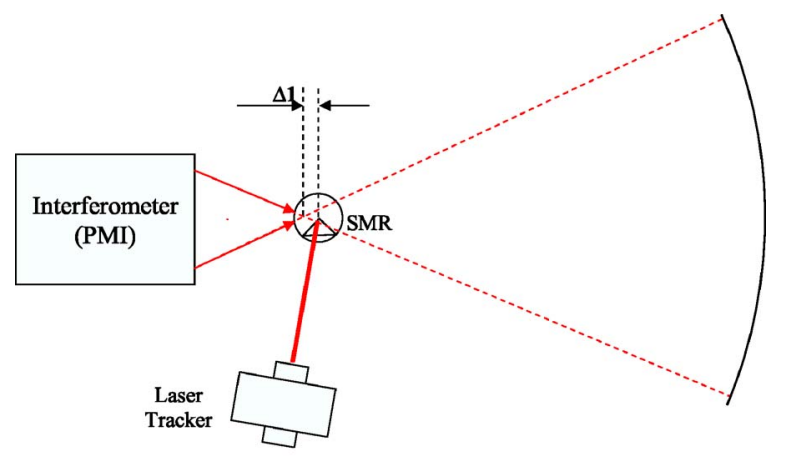

(a)

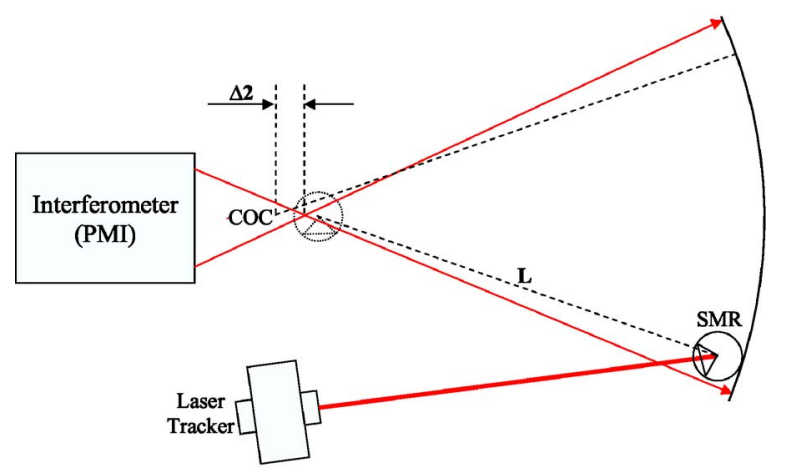

(b)

Fig. 1 Test setup to measure the ROC of a spherical mirror with a $\mathrm{PMI}$ and a laser tracker (not to scale). The quantities used in calculation of the ROC are defined here. (a) Step 1: put the SMR at the focus, record its position with the laser tracker, and measure the null cavity error with the PMI. (b) Step 2: move the SMR to the mirror surface, again record its position with the laser tracker, and measure the null cavity error with the PMI.

\section{Experiment}

We measured the ROC of a concave spherical mirror $0.5 \mathrm{~m}$ in diameter and about $2.5 \mathrm{~m}$ in ROC. The interferometer, laser tracker, and the spherical mirror were all mounted on the same optical table. Since the laser tracker measures distances with a built-in DMI, it has much higher accuracy than the angle measurement, which is done by angular encoders. To minimize the measurement error of $L$, we put the tracker close to the laser beam focus of the PMI. The tracker's laser beam was about 90 deg to the interferometer's optical axis when it tracked the ball at the focus. The position error of the tracker ball due to the angle measurement error gets coupled into the calculation of $L$, but the error was small since the distance from the tracker to the ball was small $(\sim 220 \mathrm{~mm})$. With the tracker positioned near the PMI beam focus, the tracker beam was also nearly parallel to the interferometer's axis when tracking the ball to the mirror. The angle error, in this case, mostly influences the lateral position error of the ball, which has little effect on the accuracy of the $L$ measurement. We measured distances from four points on the mirror to the focus and took the average to obtain $L$. All four points were near the edge of the mirror for convenience of mounting the SMR. The SMR can be mounted anywhere as long as it touches the test surface. The measurement results are listed in Table 1. There were about six surface tilt fringes along $-30 \mathrm{deg}$
Table 1 Results of the ROC measurement of a spherical mirror.

\begin{tabular}{|c|c|c|c|}
\hline & Item & & Value $(\mathrm{mm})$ \\
\hline \multirow{5}{*}{$\begin{array}{l}L(\text { measured } \\
\text { with a laser tracker) }\end{array}$} & Point 1 & 2480.856 & 2480.857 \\
\hline & Point 2 & 2480.852 & \\
\hline & Point 3 & 2480.859 & \\
\hline & Point 4 & 2480.861 & \\
\hline & Average & 2480.857 & \\
\hline $\begin{array}{l}\Delta 1 \text { (measured } \\
\text { with a } P M I)\end{array}$ & & & 0.155 \\
\hline $\begin{array}{l}\Delta 2 \text { (measured } \\
\text { with a PMI) }\end{array}$ & & & -0.071 \\
\hline $\begin{array}{l}r \text { (measured } \\
\text { with a micrometer) }\end{array}$ & & & 19.050 \\
\hline ROC (calculated) & & & 2499.992 \\
\hline
\end{tabular}

with the $x$ axis across the test mirror aperture during the experiment. The differences within the $L$ measurements for the four points can be partly explained by the tilt fringes.

We used a Laser Tracker II ${ }^{\mathrm{PLUS}}$ from Automated Preci-

Table 2 Uncertainty analysis for the ROC measurement listed in Table 1.

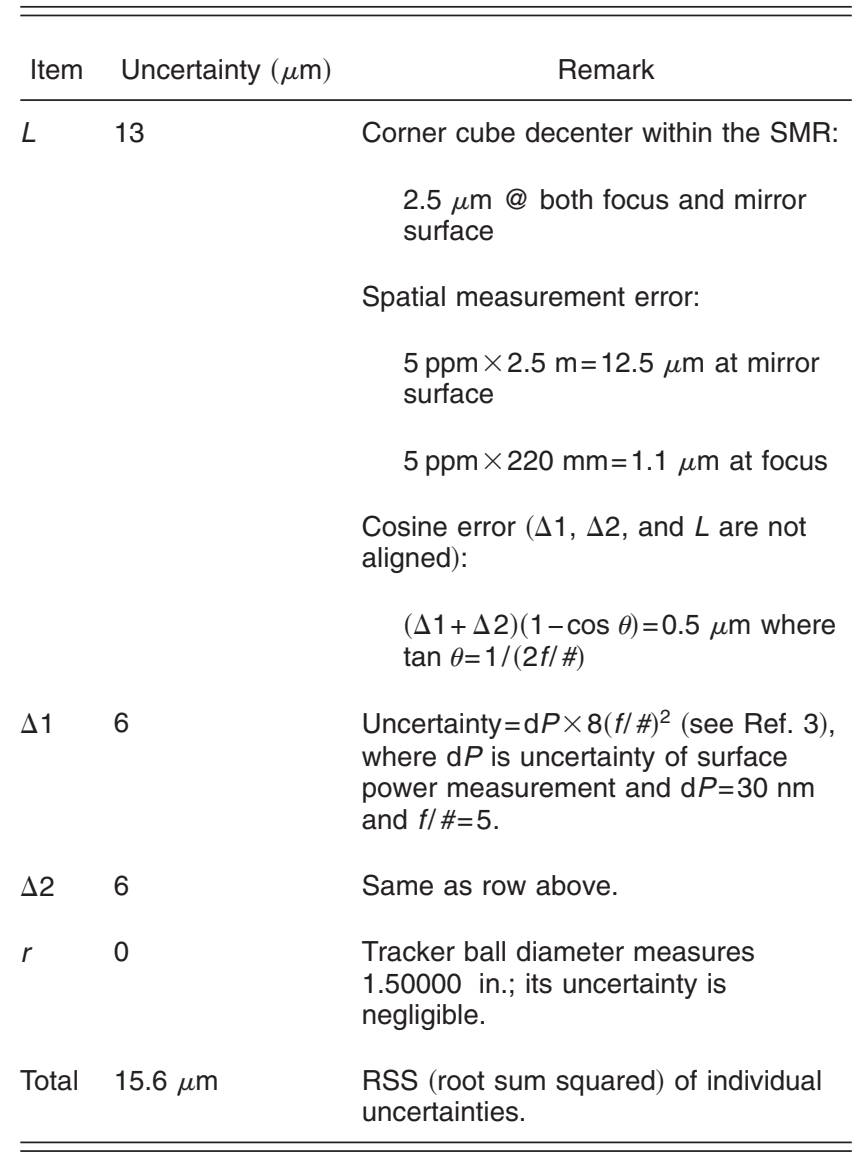


sions Inc. (see, e.g., Ref. 6). It offers 0.7-arcsec. angle measurement accuracy and $5 \mathrm{ppm}$ absolute accuracy in 3-D spatial measurements. Using these accuracies, along with the estimated errors of the PMI wavefront measurements, we performed a measurement uncertainty analysis. The analysis indicates the measurement uncertainty is less than $\pm 20 \mu \mathrm{m}$. The analysis results are summarized in Table 2 .

For comparison, we measured the same mirror with a Mitutoyo 140 series inside micrometer ${ }^{7}$ and the same PMI. We obtained an ROC of $2499.915 \mathrm{~mm}$, or $77 \mu \mathrm{m}$ less than that from the PMI and laser tracker measurement. We used a combination of six steel rods $(2+4+4+8+40+40$ in.) for this measurement, and the measurement accuracy specified by Mitutoyo is $\pm(7+n+L / 50) \mu \mathrm{m}$, where $n$ is the number of rods and $L$ is the maximum distance in millimeters. In our case, $n=6$ and $L=2500 \mathrm{~mm}$, so the specified accuracy is $\pm 63 \mu \mathrm{m}$. The room temperature was $22^{\circ} \mathrm{C}$ when the measurement was made, while the lengths of the rods are specified at $20^{\circ} \mathrm{C}$. The rods are made of tool steel with thermal expansion coefficients $18 \mathrm{ppm} /{ }^{\circ} \mathrm{C}$. The $2{ }^{\circ} \mathrm{C}$ difference caused the rods to expand by as much as $90 \mu \mathrm{m}$. After the thermal expansions of the rods were compensated, the mea- sured ROC was $2500.005 \mathrm{~mm} \pm 63 \mu \mathrm{m}$. Thus, the PMI and laser tracker measurement is within the uncertainty of the PMI and inside micrometer measurement.

\section{Conclusion}

We presented a method to use a PMI and a laser tracker to measure the ROC of a spherical mirror. The laser tracker can be placed next to the PMI, which makes the testing system more compact. During the measurements, only the tracker ball is moved from the PMI focus to the test surface and the test piece stays where it is. This is a convenient way to measure a spherical mirror, especially a concave one, with a mid to long ROC.

\section{References}

1. D. Malacara, Optical Shop Testing, pp. 460-464, Wiley, New York (1978).

2. M. C. Gerchman and G. C. Hunter, "Differential technique for accurately measuring the radius of curvature of long radius concave optical surfaces," Opt. Eng. 19(6), 843-848 (1980).

3. L. A. Selberg, "Radius measurement by interferometry," Opt. Eng. 31(9), 1961-1966 (1992).

4. A. Davies and T. L. Schmitz, "Defining the measurand in radius of curvature measurements," Proc. SPIE 5190, 134-145 (2003).

5. Y. Xiang, "Focus retrocollimated interferometry for long-radius-ofcurvature measurement," Appl. Opt. 40, 6210-6214 (2001)

6. www.apisensor.com.

7. See http://www.mitutoyo.com/catalog/pdf/N-08-09.pdf. 